\title{
NLRP3/Caspase-1 Pathway-Induced Pyroptosis Mediated Cognitive Deficits in a Mouse Model of Sepsis-Associated Encephalopathy
}

\author{
Qun Fu, ${ }^{1,2}$ Jing Wu, ${ }^{3,4}$ Xiao-Yan Zhou, ${ }^{1}$ Mu-Huo Ji, ${ }^{5}$ Qing-Hong Mao, ${ }^{2}$ Qing Li, $^{2}$ \\ Man-Man Zong, ${ }^{1}$ Zhi-Qiang Zhou, ${ }^{1,6}$ and Jian-Jun Yang ${ }^{4,5,6}$
}

\begin{abstract}
Sepsis-associated encephalopathy (SAE) is a common complication that leads to long-term cognitive impairments and increased mortality in sepsis survivors. The mechanisms underlying this complication remain unclear and an effective intervention is lacking. Accumulating evidence suggests the nucleotide-binding domain-like receptor protein3 (NLRP3)/caspase-1 pathway is involved in several neurodegenerative diseases. Thus, we hypothesized that the NLRP3/caspase-1 pathway is involved in NLRP3-mediated pyroptosis, maturation and release of inflammatory cytokines, and cognitive deficits in SAE. We used the NLRP3 inhibitor MCC950 and the caspase-1 inhibitor Ac-YVAD-CMK to study the role of the NLRP3/caspase-1 pathway in pyroptosis and cognitive deficits in a mouse model of SAE. Mice were randomly assigned to one of six groups: sham+saline, sham+MCC950, sham+Ac-YVAD-CMK, cecal ligation and puncture (CLP)+saline, CLP+MCC950, and CLP+Ac-YVAD-CMK. Surviving mice underwent behavioral tests or had hippocampal tissues collected for histochemical analysis and biochemical assays. Our results show that CLP-induced hippocampus-dependent memory deficits are accompanied by increased NLRP3 and caspase-1 positive cells, and augmented protein levels of NLRP3, caspase-1, gasdermin-D, and pro-inflammatory cytokines in the hippocampus. In addition, administration of MCC950 or Ac-YVAD-CMK rescues cognitive deficits and ameliorates increased hippocampal NLRP3-mediated neuronal pyroptosis and pro-inflammatory cytokines. Our results suggest that the NLRP3/caspase-1 pathway-induced pyroptosis mediates cognitive deficits in a mouse model of SAE.
\end{abstract}

KEY WORDS: NLRP3; caspase-1; pyroptosis; pro-inflammatory cytokine; cognitive impairment.

Qun Fu and Jing Wu contributed equally to this work.

${ }^{1}$ Department of Anesthesiology, Jinling Hospital, Medical College of Nanjing Medical University, Nanjing, 210002, China

${ }^{2}$ Department of Anesthesiology, Affiliated Hospital of Integrated Traditional Chinese and Western Medicine, Nanjing University of Chinese Medicine, Nanjing, 210028, China

${ }^{3}$ Jiangsu Key Laboratory of Molecular Medicine, Medical School of Nanjing University, Nanjing, 210093, China

\footnotetext{
${ }^{4}$ Department of Anesthesiology, The first Affiliated Hospital of Zhengzhou University, Zhengzhou, 450000, China

${ }^{5}$ Department of Anesthesiology, Zhongda Hospital, Medical School, Southeast University, Nanjing, 210009, China

${ }^{6}$ To whom correspondence should be addressed to Zhi-Qiang Zhou at Department of Anesthesiology, Jinling Hospital, Medical College of Nanjing Medical University, Nanjing, 210002, China. E-mail: zq_zhou@163.com; and Jian-Jun Yang at Department of Anesthesiology, The first Affiliated Hospital of Zhengzhou University, Zhengzhou, 450000, China. E-mail: yjyangjj@126.com
} 


\section{INTRODUCTION}

Sepsis-associated encephalopathy (SAE), which is characterized by long-term cognitive impairments and psychiatric diseases in sepsis survivors, is associated with increased morbidity and mortality [1-4]. Several mechanisms are involved in the pathogenesis of SAE, such as endothelial activation, disturbance of the blood-brain barrier, oxidative damage, neurotransmission disturbances, altered brain signaling, and neuronal apoptosis [5]. There is accumulating evidence that neuroinflammation plays a key role in the development of SAE $[1,6]$. Nonetheless, the mechanisms by which sepsis induces overactivated neuroinflammation have yet to be elucidated.

Pyroptosis is an inflammatory form of programmed cell death and plays a protective role in host defense during infection. This kind of cell death is characterized by cell swelling, lysis, and release of cytoplasmic content. The initiation of pyroptosis leads to pore-mediated cell lysis and IL-1 $\beta$ secretion [7]. However, excessive pyroptosis is harmful to normal tissues and cells. To avoid host organism damage, pyroptosis is tightly regulated by the activation of inflammatory caspases, such as caspase- 1 , caspase- 4 , caspase- 5 , and caspase- 11 . These caspases found in the canonical and non-canonical inflammasome signaling pathways lead to inflammatory responses [8-10]. However, it remains unclear how these caspases initiate pyroptosis.

The canonical inflammasome pathway is triggered by various cytoplasmic sensor proteins that recognize inflammatory agents and multiple pathogens and recruit procaspase-1 monomers by the apoptosis-associated specklike protein containing a CARD (ASC) and activate caspase-1 through dimerization. The non-canonical inflammasome pathway is activated by lipopolysaccharide molecules in the cytoplasm of infected cells [11]. The nucleotide-binding domain-like receptor protein 3 (NLRP3) inflammasome is an intracellular protein complex that plays a crucial role in innate immune sensing. Moreover, activation of NLRP3 leads to the maturation of caspase-1, which mediates pyroptosis [12] and regulates the cleavage and maturation of pro-inflammatory cytokines, such as IL-1 $\beta$ and IL-18 [13]. However, how much pyroptosis and increased inflammatory cytokines are involved in the cognitive impairments that occur during SAE remains unknown.

In light of these findings, we hypothesize that sepsis triggers NLRP3-mediated pyroptosis, neuro-inflammatory responses, and cognitive deficits through the NLRP3/ caspase-1 pathway, and that administration of the NLRP3 inhibitor MCC950 and the caspase-1 inhibitor Ac-YVADCMK could attenuate hippocampal neuronal pyroptosis and neuroinflammation, reducing long-term cognitive deficits and improving survival rates.

\section{MATERIALS AND METHODS}

\section{Animals}

One hundred and seventy-four C57BL/6 male mice aged 4 months were purchased from the Animal Center of Jinling Clinical Medical College at Nanjing Medical University, Nanjing, China. All experimental procedures in this study were performed according to the Guidelines for the Care and Use of Laboratory Animals from the National Institutes of Health, USA. Experiments began after mice had acclimated to the environment for 2 weeks. Mice were housed in groups of five individuals per cage with a $12: 12 \mathrm{~h}$ light:dark cycle at a temperature of $23-25^{\circ} \mathrm{C}$ with food and water available ad libitum.

\section{Surgical Procedures}

Mice were subjected to cecal ligation and puncture (CLP) as previously described [14]. Briefly, mice were intraperitoneally anesthetized using $2 \%$ sodium pentobarbital (40 mg/kg; Sigma Chemical Co, St. Louis, MO, USA). Under aseptic conditions, the cecum was carefully isolated and then ligated with 4.0 silk below the ileocecal junction, approximately $1.2 \mathrm{~cm}$ from the distal end. The cecum was then perforated twice with a sterile 22-gauge needle and gently squeezed to extrude fecal contents into the peritoneal cavity. After that, the cecum was returned to the peritoneal cavity and the laparotomy incision closed with 4.0 silk sutures. Mice subjected to sham operation had the cecum exposed in the same way as for CLP but it was neither ligated nor punctured. Mice were revived immediately after surgery by administering regular saline $(30 \mathrm{ml} /$ $\mathrm{kg}$ ) subcutaneously and returned to their cages.

\section{Drug Administrations}

Mice were randomly divided into one of the following six groups: sham+saline $(n=18)$, sham+MCC950 $(n=$ 18), sham+Ac-YVAD-CMK $(n=18), \mathrm{CLP}+$ saline $(n=$ 40), CLP+MCC950 $(n=40)$, and CLP+Ac-YVAD-CMK $(n=40)$. Saline, MCC950 (10 mg/kg, China Peptides Co. Ltd., China) [15], or Ac-YVAD-CMK (100 $\mu$ g per mouse, Cayman Chemical Company, USA) [16] was intraperitoneally injected $30 \mathrm{~min}$ before surgery and on days 1, 2, 4, 
and 6 after surgery. Seven days after surgery, six mice in each group were deeply anesthetized using $2 \%$ sodium pentobarbital $(60 \mathrm{mg} / \mathrm{kg})$ and decapitated. The brain was rapidly removed and separated into two halves for histochemical analysis and biochemical assays. The remaining mice in each group were used in behavioral tests 2 weeks after surgery.

\section{Hematoxylin and Eosin (HE) Staining}

Half of each mouse brain harvested ( $n=6$ for each group) underwent HE staining and immunohistochemical analysis. Brain tissues were immersed in $4 \%$ paraformaldehyde and embedded using paraffin. The tissues were sliced into 4-mm sections until use.

HE staining was performed as follows: hematoxylin staining for $5 \mathrm{~min}$; $75 \%$ hydrochloric acid alcohol solution for $30 \mathrm{~s}$ decoloring; eosin staining for $5 \mathrm{~min}$; and, $90 \%$ ethanol for $35 \mathrm{~s}$ decoloring. Normal neurons have a relatively large cell body that is rich in cytoplasm with one or two big round nuclei, while damaged cells show shrunken cell bodies, pyknotic nuclei, dark cytoplasm, and many empty vesicles. Hippocampal neuronal damage was evaluated using a standard semi-quantitative scale [17]. In brief, grade 0 , no damage to any hippocampal subregion; grade 1, scattered neurons are damaged in the CA1 subregion; grade 2, moderate numbers of damaged neurons in the CA1 subregion $(<50 \%$ neurons damaged); grade 3 , severe damage ( $>50 \%$ of cells affected) to pyramidal cells in the CA1 subregion; and grade 4, extensive cell damage in all hippocampal regions. Four random high-power $(\times$ 400) visual fields from each brain slice were checked. Evaluation of cells with nuclear pyknosis and morphologic abnormality was performed by two pathologists blind to the treatment groups.

\section{Immunohistochemistry}

Paraffin sections were then deparaffinized and hydrated using the following steps: $10 \mathrm{~min}$ in xylene twice; 5,10 , 10 , and $10 \mathrm{~min}$ in $100 \%, 95 \%, 85 \%$, and $70 \%$ ethanol, respectively; and $5 \mathrm{~min}$ in PBS at room temperature repeated three times. Antigen retrieval was achieved by boiling the sections in $10 \mathrm{mM}$ sodium citrate for $10 \mathrm{~min}$ in a microwave oven. Sections were then washed with PBS three times and treated with $3 \% \mathrm{H}_{2} \mathrm{O}_{2}$-methanol for $15 \mathrm{~min}$. Immunostaining was performed by incubation with antibodies against NLRP3 (1:100; Servicebio Technology Co. Ltd., Wuhan, China) and caspase-1 (1:100; Servicebio Technology Co. Ltd., Wuhan, China); cells with brownish yellow cytoplasm were recorded as positive cells. The numbers of NLRP3 and caspase-1 immunoreactive cells in the hippocampal CA1 region were measured by an investigator blind to group assignment.

\section{Western Blotting Analysis}

The hippocampus was harvested from the second brain half from each animal ( $n=6$ for each group) and homogenized for biochemical assays. Proteins from each hippocampus were electrophoretically separated and blotted onto nitrocellulose membranes. Protein levels were estimated via incubation against antibodies of NLRP3 (1:600; Servicebio Technology Co. Ltd.), ASC (1:200; Santa Cruz, USA), cleaved caspase-1 (1:500; Servicebio Technology Co. Ltd.), gasdermin-D (GSDMD, 1:500; Biorbyt, UK), IL-1 $\beta$ (1:200; Santa Cruz, USA), IL-18 (1:200; Santa Cruz, USA), and $\beta$ actin (1:5000; Bioworld, USA). Protein bands were visualized via enhanced chemiluminescence and quantified using ImageQuant software (Syngene).

\section{Enzyme-Linked Immunosorbent Assay}

Hippocampal IL-1 $\beta$ and IL-18 levels were quantified using an enzyme-linked immunosorbent assay (ELISA) kit according to the manufacturer's directions (Servicebio Technology Co. Ltd).

\section{OpenField Test}

Behavioral tests were conducted on surviving mice 2 weeks after surgery in a sound-isolated room by a single investigator that was blind to treatment group assignment. All tests were conducted between 14:00 and 17:00 h. Locomotor and exploratory activities of mice were measured in an open-field apparatus [18]. A mouse was gently placed in the center of a white plastic chamber $(50 \times 50 \times 30 \mathrm{~cm})$ for $5 \mathrm{~min}$ and activities were automatically recorded using a video tracking system (XR-XZ301, Shanghai Xinruan Soft Information Technology Co. Ltd., Shanghai, China). The apparatus was cleaned with $75 \%$ alcohol between tests to remove any odor cues.

\section{Fear Conditioning Test}

Two hours after the open-field test, mice were trained for a fear conditioning test, as described previously [18]. Each mouse was gently placed into a conditioning chamber (XRXC404, Shanghai Soft Maze Information Technology Co., Ltd., Shanghai, China) and allowed to acclimate for $3 \mathrm{~min}$. A 30 -s tone $(75 \mathrm{~dB}, 3 \mathrm{kHz})$ was then delivered followed by a 2 -s foot shock $(0.75 \mathrm{~mA})$. The mouse was 
kept in the chamber for another $30 \mathrm{~s}$ and then returned to its home cage. A context test to evaluate hippocampusdependent memory was performed $24 \mathrm{~h}$ after training. Each mouse was returned to the same test chamber for 5 min without any stimulation. After the context test, each mouse was placed for $390 \mathrm{~s}$ in a novel chamber altered in shape, color, and smell. The same tone was presented for another $3 \mathrm{~min}$ without the foot shock to evaluate hippocampus-independent memory. Cognitive deficits in the test was assessed by measuring the amount of time the mouse demonstrated "freezing behavior," which is defined as a completely immobile posture except for respiratory efforts. Freezing behavior was automatically recorded by a video tracking system.

\section{Statistical Analysis}

Statistical analyses were done using the software Statistical Product for Social Sciences (SPSS; version 16.0, Chicago, IL, USA) and data plotted using GraphPad Prism 5.0 Software (San Diego, CA, USA). Survival rate was assessed by the Kaplan-Meier method and compared among treatment groups using the log-rank test. Differences among groups were tested using a one-way analysis of variance followed by Tukey's tests. Data are expressed as mean \pm SEM. A $p$ value $<0.05$ was considered statistically significant.

\section{RESULTS}

\section{MCC950 or Ac-YVAD-CMK Improved Survival Rate of CLP Mice}

Mouse mortality increased for 7 days after CLP, confirming the results of our previous study [14]. In this study, survival rate increased with administration of intraperitoneal MCC950 (77.5\%) or Ac-YVAD-CMK (75\%), compared to the CLP + saline group (52.5\%; Fig. 1). This indicates that MCC950 and Ac-YVAD-CMK improve the survival rate of CLP mice.

\section{MCC950 or Ac-YVAD-CMK Attenuated Hippocampus-Dependent Memory Impairments in SAE Mice}

Mice recover with no signs of infection or motor alterations by 10 days post-CLP [19]. Thus, behavior tests were done 2 weeks after surgery. The total distance moved and time spent in the center of the arena were similar among all six groups (Fig. 2a, b).

A fear conditioning test was used to evaluate whether CLP-induced long-term memory impairments were present $24 \mathrm{~h}$ after training. The CLP+saline group had a shorter freezing time of context test than the sham+saline group, indicating the presence of a sepsis-induced

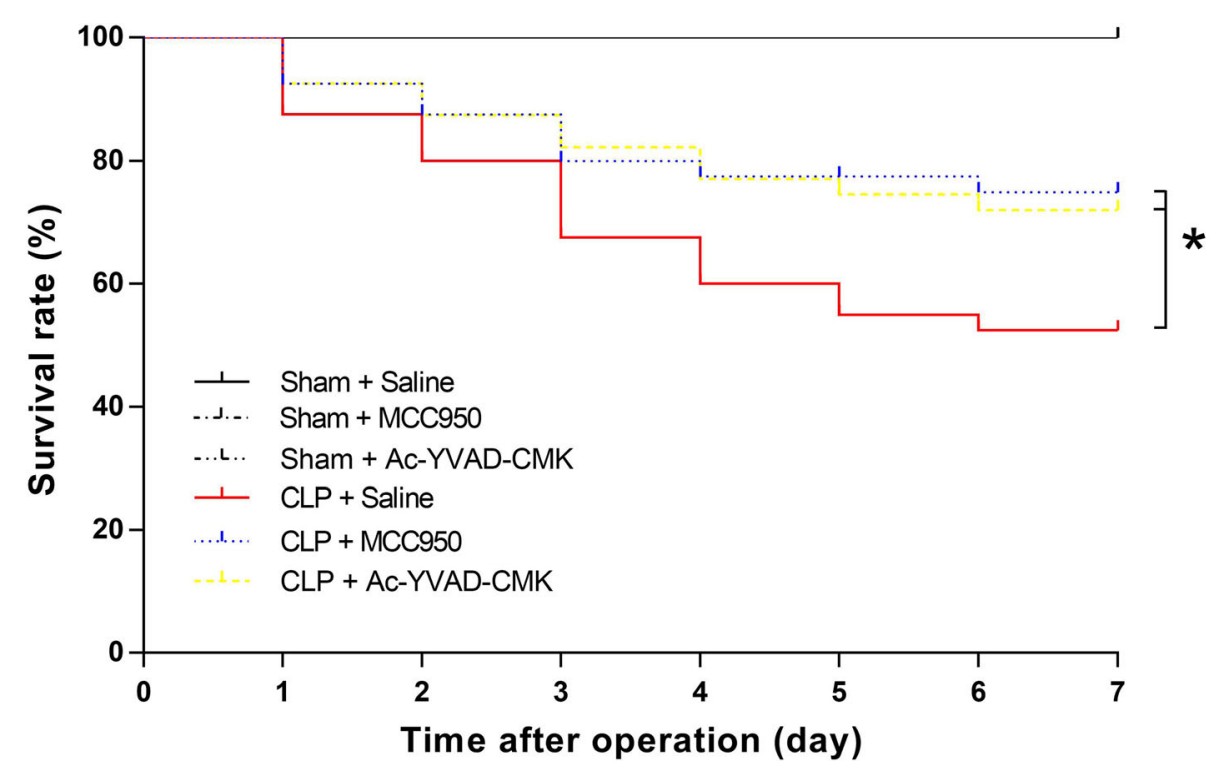

Fig. 1. MCC950 or Ac-YVAD-CMK improved survival rate in the first 7 days after CLP. Mice were randomly divided into six groups: sham+saline, sham+ MCC950, sham+Ac-YVAD-CMK, CLP+saline, CLP+MCC950, and CLP+Ac-YVAD-CMK. Normal saline (0.25 ml per mouse), MCC950 (10 mg/kg), or Ac-YVAD-CMK (100 $\mu \mathrm{g}$ per mouse) was administered to mice intraperitoneally $30 \mathrm{~min}$ before surgery and on days 1, 2, 4, and 6 after surgery. Survival rate to day 7 was evaluated ( $n=18-40$ mice/group). The asterisk indicates $P<0.05$ versus the CLP+saline group. 
hippocampus-dependent long-term memory deficit. However, this memory deficit was ameliorated by administration of MCC950 or Ac-YVAD-CMK (Fig. 2c). No differences were observed in freezing time of the cue test among the six groups (Fig. 2d), indicating that hippocampus-independent memory deficit was not found in the SAE mice. Our results suggest that MCC950 and Ac-YVAD-CMK have a therapeutic effect on CLP-induced cognitive deficits.
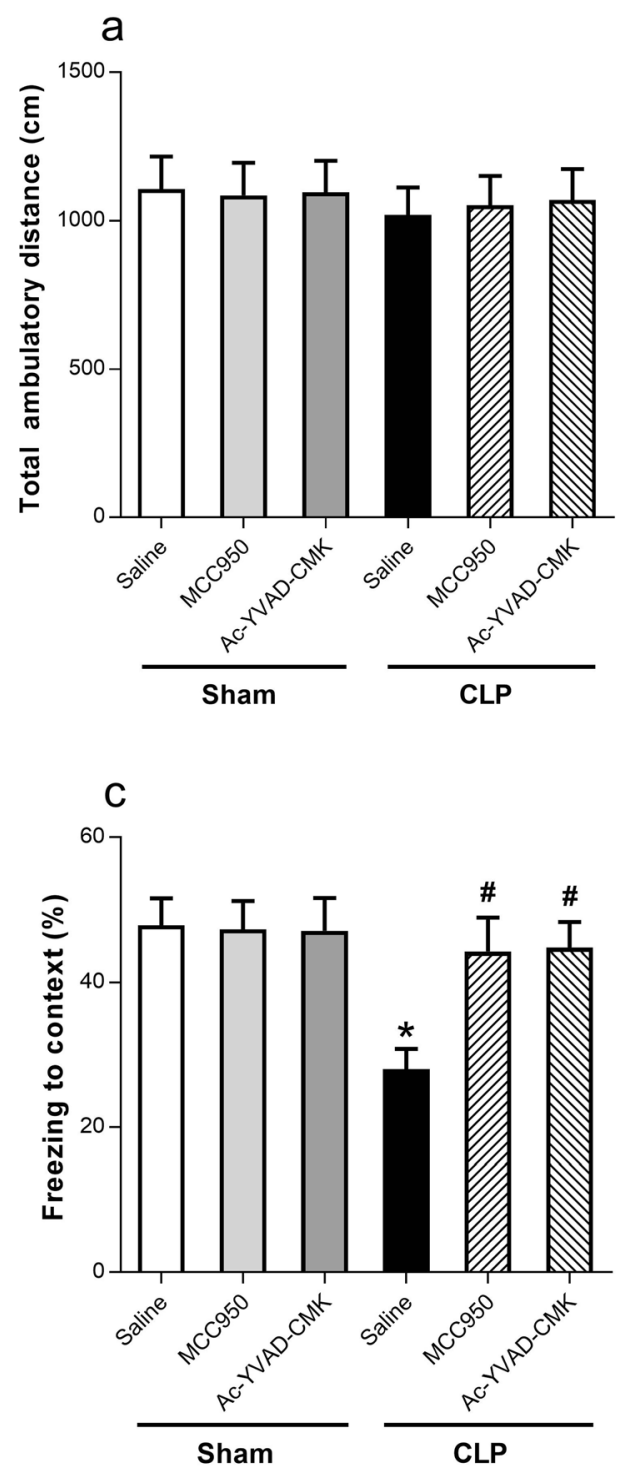

\section{MCC950 or Ac-YVAD-CMK Rescued Morphological Damages of Hippocampus in SAE Mice}

To evaluate sepsis-induced histological damage and the neuroprotective effects of MCC950 and AcYVAD-CMK, we did HE staining on brain sections of the hippocampus CA1 region 7 days after surgery. No abnormalities in pyramidal cell morphology were observed in the sham-operated groups. The CLP+saline
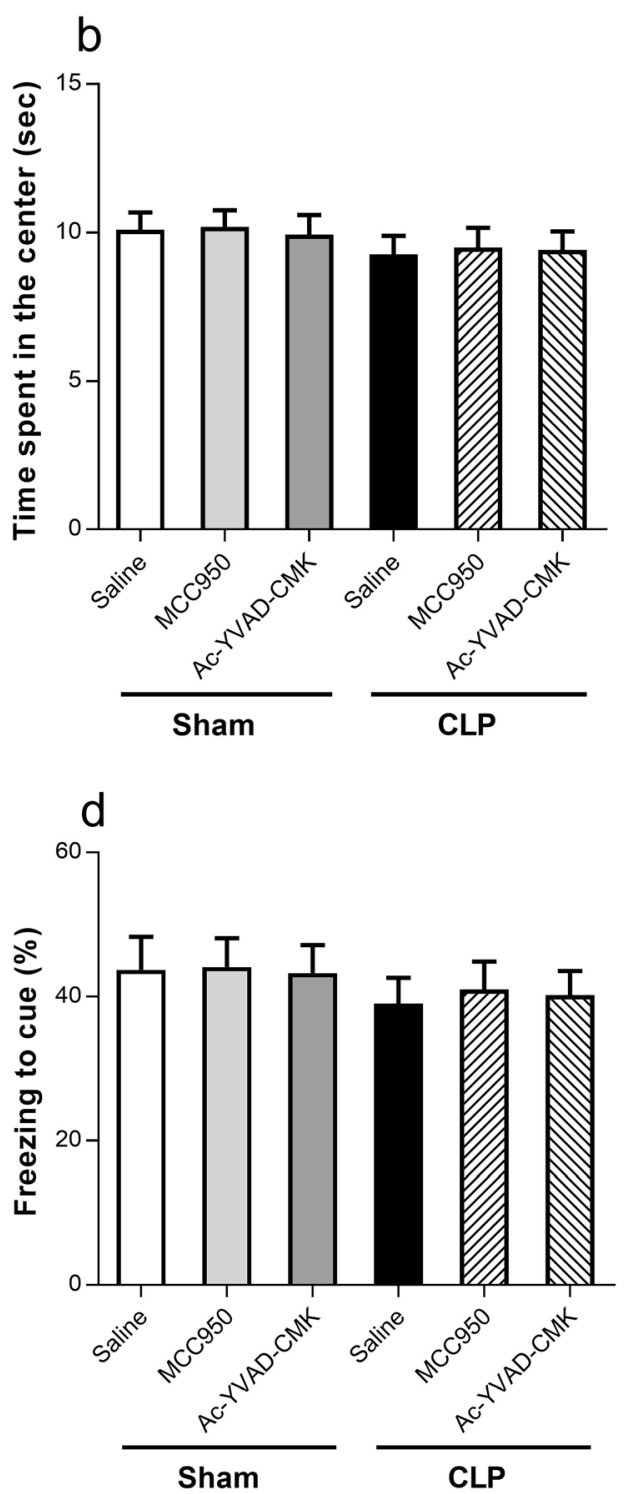

Fig. 2. MCC950 or Ac-YVAD-CMK reversed CLP-induced learning and memory impairments. a Total ambulatory distance. b Time spent in the center. c The contextual fear conditioning test. $\mathbf{d}$ The cued fear conditioning test. Data are presented as the mean \pm SEM ( $n=12-24$ mice/group). The asterisk indicates $P<0.05$ versus the sham+saline group; the pound sign, $P<0.05$ versus the CLP+saline group. For the procedures of the open-field test and fear conditioning test, see "MATERIALS AND METHODS." 
group showed the most shrunken cell bodies and largest amount of nuclear pyknosis (Fig. 3). Moreover, mice given MCC950 or Ac-YVAD-CMK showed significantly fewer abnormal neurons and lower scores of neuronal damage compared to the CLP+saline group (Fig. 3). This indicates that MCC950 or Ac-YVAD-
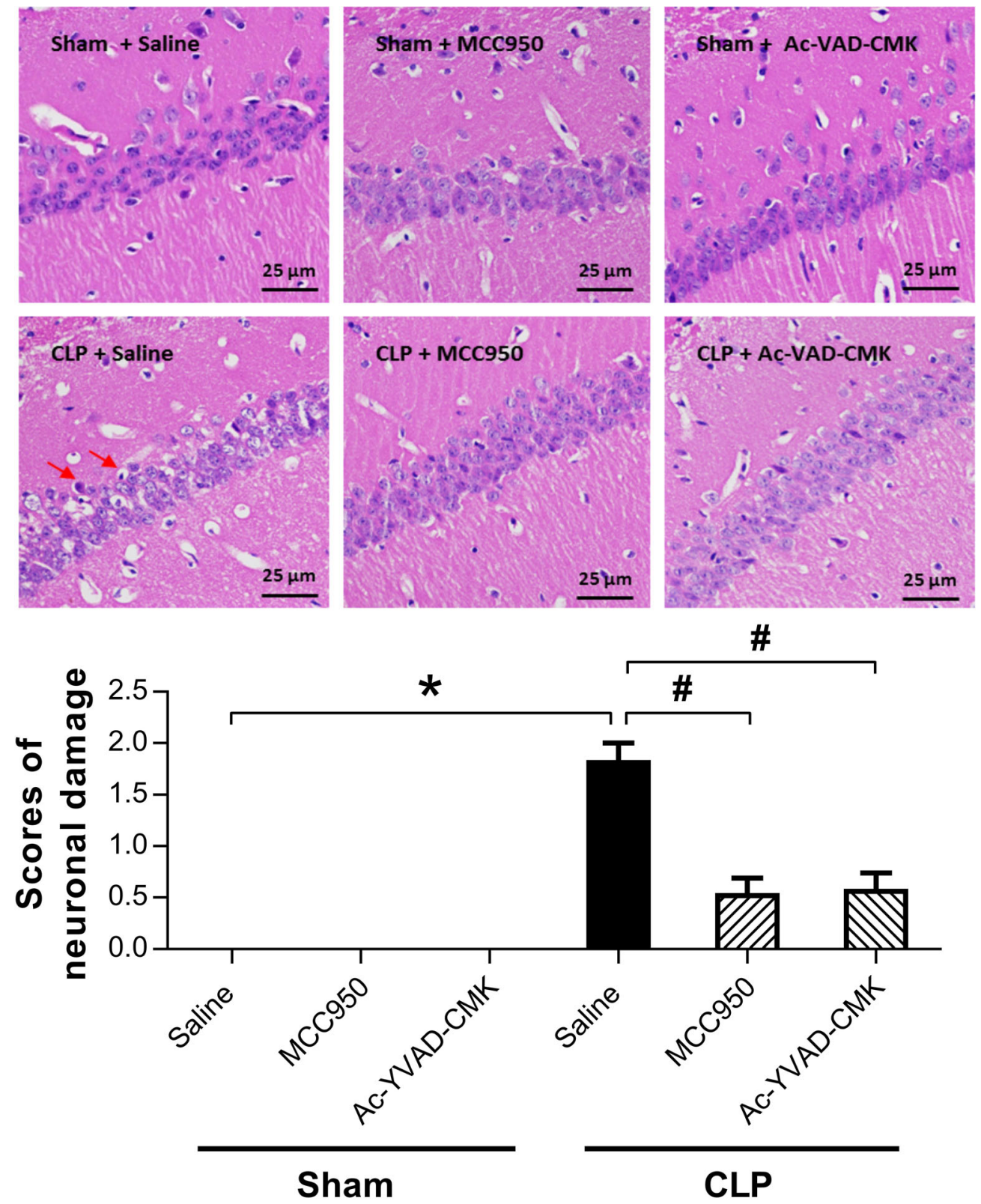

Fig. 3. MCC950 or Ac-YVAD-CMK reduced CA1 neuronal damages in the hippocampus of SAE mice. a Representative pictures of HE staining showing morphologic abnormality of pyramidal cells in the hippocampus CA1 region. Pyramidal cells had an orderly arrangement and had complete cell structure in the sham groups; in CLP+saline group, cells had a disorderly arrangement, with the cytoplasm and nucleolus dyed deeply indicating nuclear pyknosis (indicated by arrows). The number of degenerate cells in the CLP+MCC950 group and CLP+Ac-YVAD-CMK groups was reduced relative to the CLP+ saline group. Scale bar indicates $25 \mu \mathrm{m}$. b Semi-quantitative analysis shows significant reduction in hippocampal neuronal damage for mice treated with MCC950 or Ac-YVAD-CMK compared to mice treated with saline after CLP. Data are presented as the mean \pm SEM ( $n=6$ mice/group). The asterisk indicates $P<0.05$ versus the sham+saline group; the pound sign, $P<0.05$ versus the CLP+saline group. 
CMK can rescue hippocampal neurons from CLPinduced morphological damage.

\section{MCC950 or Ac-YVAD-CMK Inhibited the Activation of NLRP3/Caspase-1 Pathway in the Hippocampus of SAE Mice}

The NLRP3 inflammasome, a component of the inflammatory process, is highly expressed in various neurodegenerative disorders [20-22]. CLP induces the activation of NLRP3 inflammasome in the hippocampus of SAE mice [14]. To explore whether MCC950 or Ac-YVADCMK inhibits the activation of the NLRP3/caspase-1 pathway, we measured levels of two markers of the inflammasome, NLRP3 and ASC, that may further activate caspase-1 [12, 23]. Our results showed that the protein levels of NLRP3 and cleaved caspase-1 were higher at 7 days post-CLP, and that this increase did not occur when MCC950 was administered (Fig. 4). Ac-YVAD-CMK treatment rescued the increase of cleaved caspase-1 but not that of NLRP3 (Fig. 4). No difference was observed in the levels of ASC among the six groups (Fig. 4). Immunohistochemical analysis revealed a significant increase in the number of cells positive for NLRP3 and caspase- 1 in the CA1 region of the mouse brain at 7 days post-CLP, and that this was reversed by administration of MCC950 (Fig. 5). Ac-YVAD-CMK treatment rescued the increase of caspase-1, but not that of NLRP3 (Fig. 6). These results suggest the CLP-induced activation of NLRP3/caspase-1 pathway is inhibited by the specific inhibitors MCC950 and Ac-YVAD-CMK.

\section{MCC950 or Ac-YVAD-CMK Attenuated NLRP3/ Caspase-1-Mediated Pyroptosis and Inflammatory Cytokines in SAE Mice}

The progressive dysfunction and death of neurons provide a molecular and cellular basis for memory deficits in SAE. Thus, we further investigated the role of NLRP3/ caspase-1-mediated pyroptosis in cognitive deficit of SAE
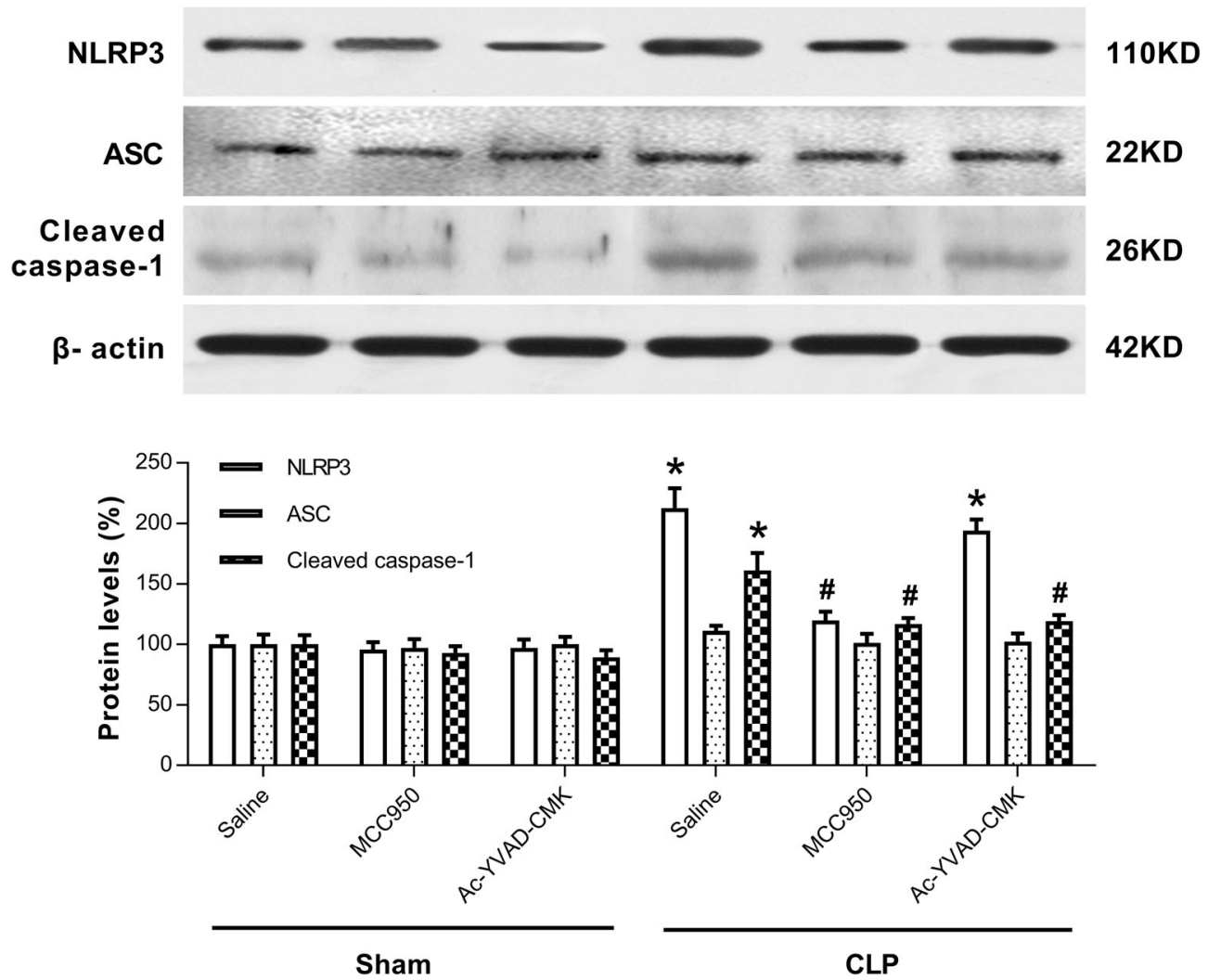

Fig. 4. MCC950 or Ac-YVAD-CMK inhibited the activation of NLRP3/caspase-1 pathway in the hippocampus of SAE mice. Representative Western blot and quantitative analysis of protein levels of NLRP3, ASC, and caspase-1 in hippocampal tissues. Data are shown as mean $\pm \operatorname{SEM}(n=6 \mathrm{mice} / \mathrm{group})$. The asterisk indicates $P<0.05$ versus the sham+saline group; the pound sign, $P<0.05$ versus the CLP+saline group. 

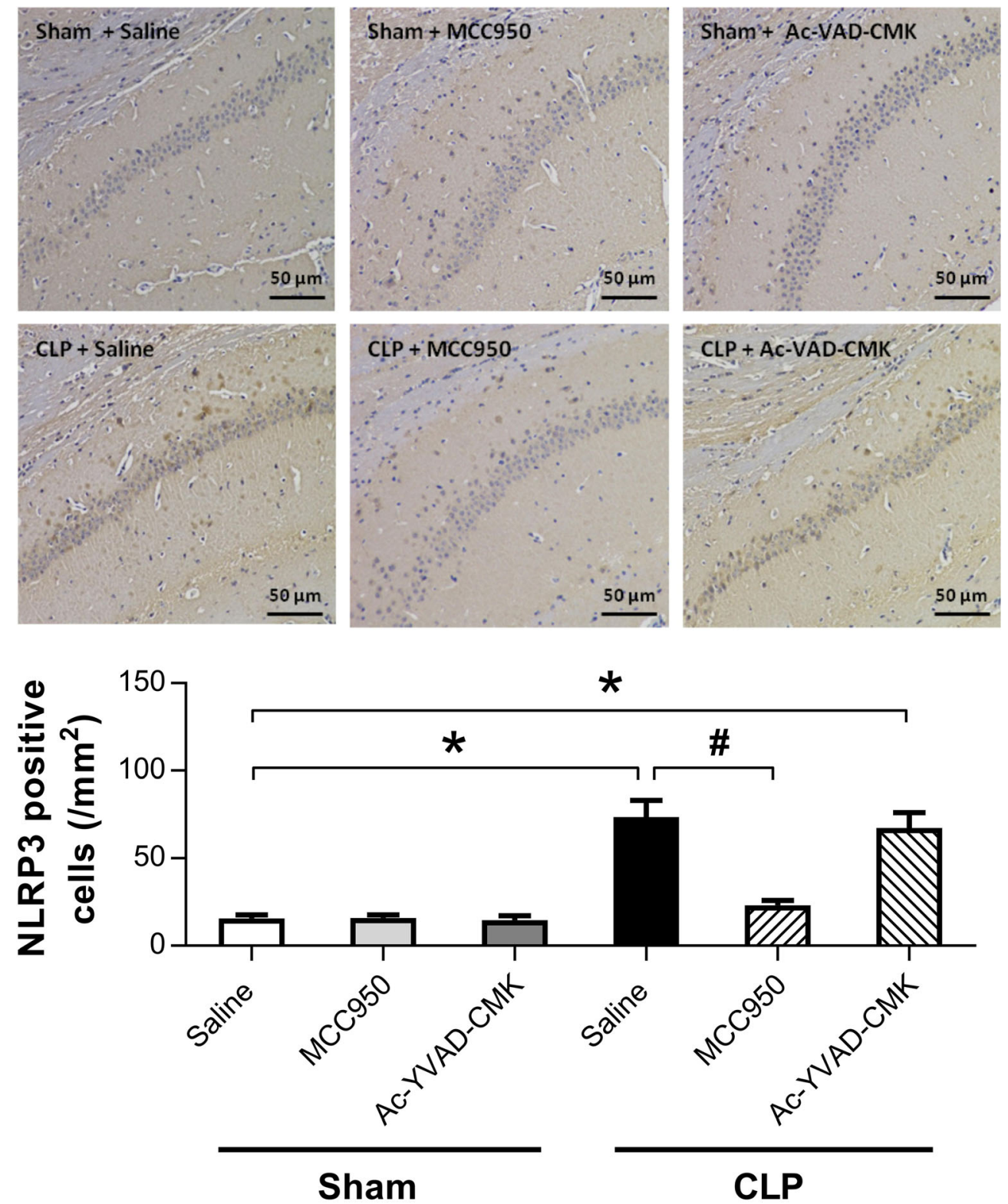

Fig. 5. Effects of MCC 950 and Ac-YVAD-CMK on the number of NLRP3 positive cells in the hippocampal CA1 region. Representative images of NLRP3 immunohistochemical (IHC) staining in the hippocampal CA1 region. Cells with brownish-yellow cytoplasm are positive for NLRP3. Scale bar indicates $50 \mu \mathrm{m}$. Lower panel presents statistics for the six experimental groups. Data are shown as mean $\pm \mathrm{SEM}$ ( $n=6$ mice/group). The asterisk indicates $P<0.05$ versus the sham+saline group; the pound sign, $P<0.05$ versus the CLP+saline group.

mice [24-26]. GSDMD is identified as the executioner of pyroptosis [27-29]. We measured the protein level of GSDMD, the specific biomarker for pyroptosis. Our result showed that CLP induced an increase of GSDMD and that administration of MCC950 or Ac-YVAD-CMK reversed this increase, implying that the NLRP3/caspase-1 pathway mediates pyroptosis after CLP (Fig. 7a).
Caspase- 1 regulates the cleavage and maturation of the downstream inflammatory cytokines IL- $1 \beta$ and IL18. The CLP inflammatory process is associated with production of pro-inflammatory IL- $1 \beta$ and IL-18. To evaluate the effect of MCC950 and Ac-YVAD-CMK on hippocampal inflammation response, we measured the levels of IL- $1 \beta$ and IL- 18 by Western blot and ELISA 

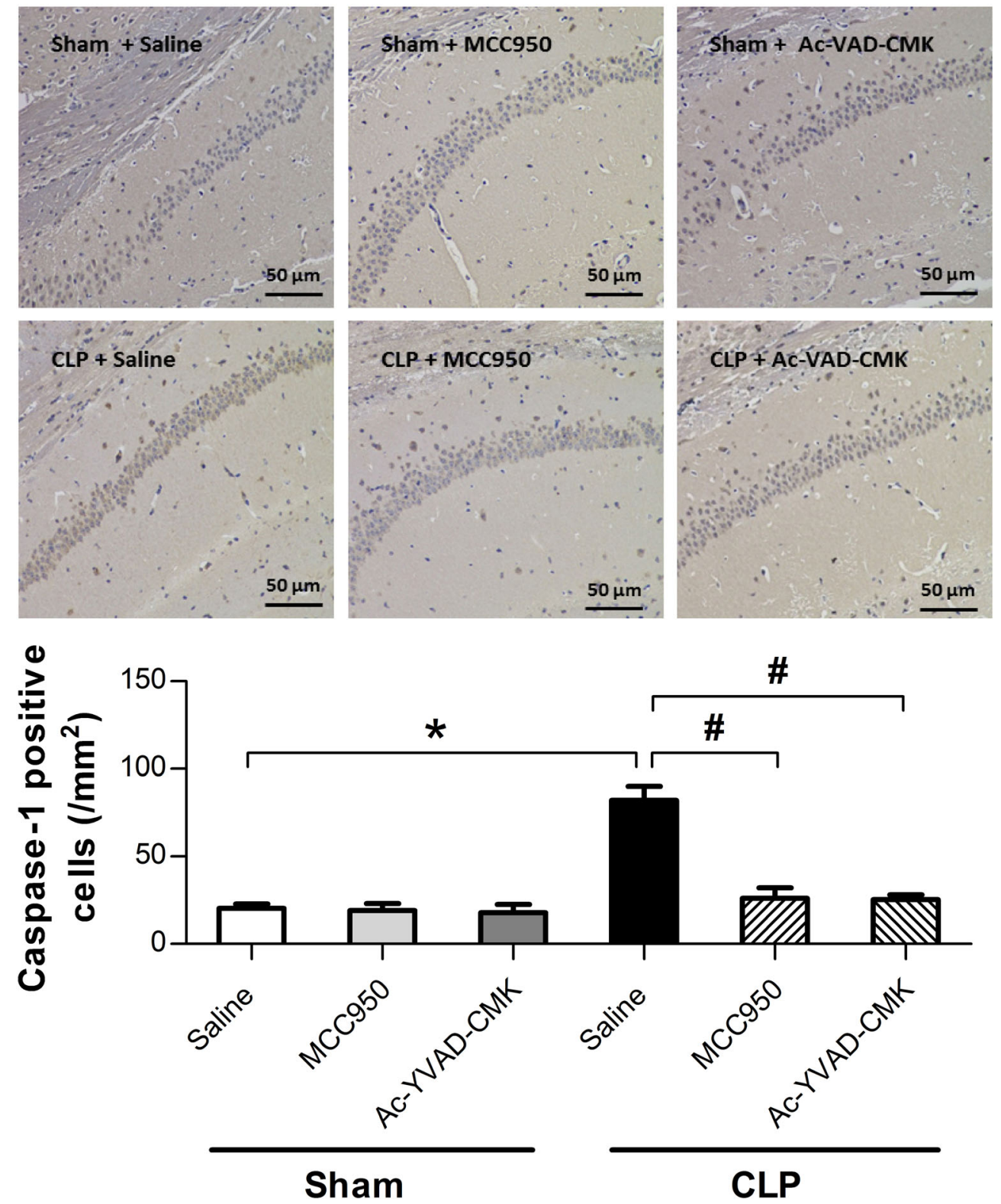

Fig. 6. Effects of MCC950 and Ac-YVAD-CMK on the number of caspase-1 positive cells in the hippocampal CA1 region. Representative images of caspase-1 immunohistochemical (IHC) staining in the hippocampal CA1 region. Cells with brownish-yellow cytoplasm are positive for caspase-1. Scale bar indicates $50 \mu \mathrm{m}$. Lower panel presents statistics for the six experimental groups. Data are shown as mean $\pm \operatorname{SEM}(n=6$ mice/group). The asterisk indicates $P$ $<0.05$ versus the sham+saline group; the pound sign, $P<0.05$ versus the CLP+saline group.

in SAE mice. Levels of IL-1 $\beta$ and IL-18 were significantly higher in the CLP+saline group compared to those in the sham+saline group, and administration of MCC950 or Ac-YVAD-CMK reversed this increase (Fig. 7a, b). Thus, inhibition of the NLRP3/caspase-1 pathway appears to alleviate inflammatory responses.

\section{DISCUSSION}

In this study, we show that administration of either the NLRP3-inhibitor MCC950 or the caspase-1 inhibitor Ac-YVAD-CMK reduces mortality, reverses cognitive impairments, and rescues neuronal damages 
a
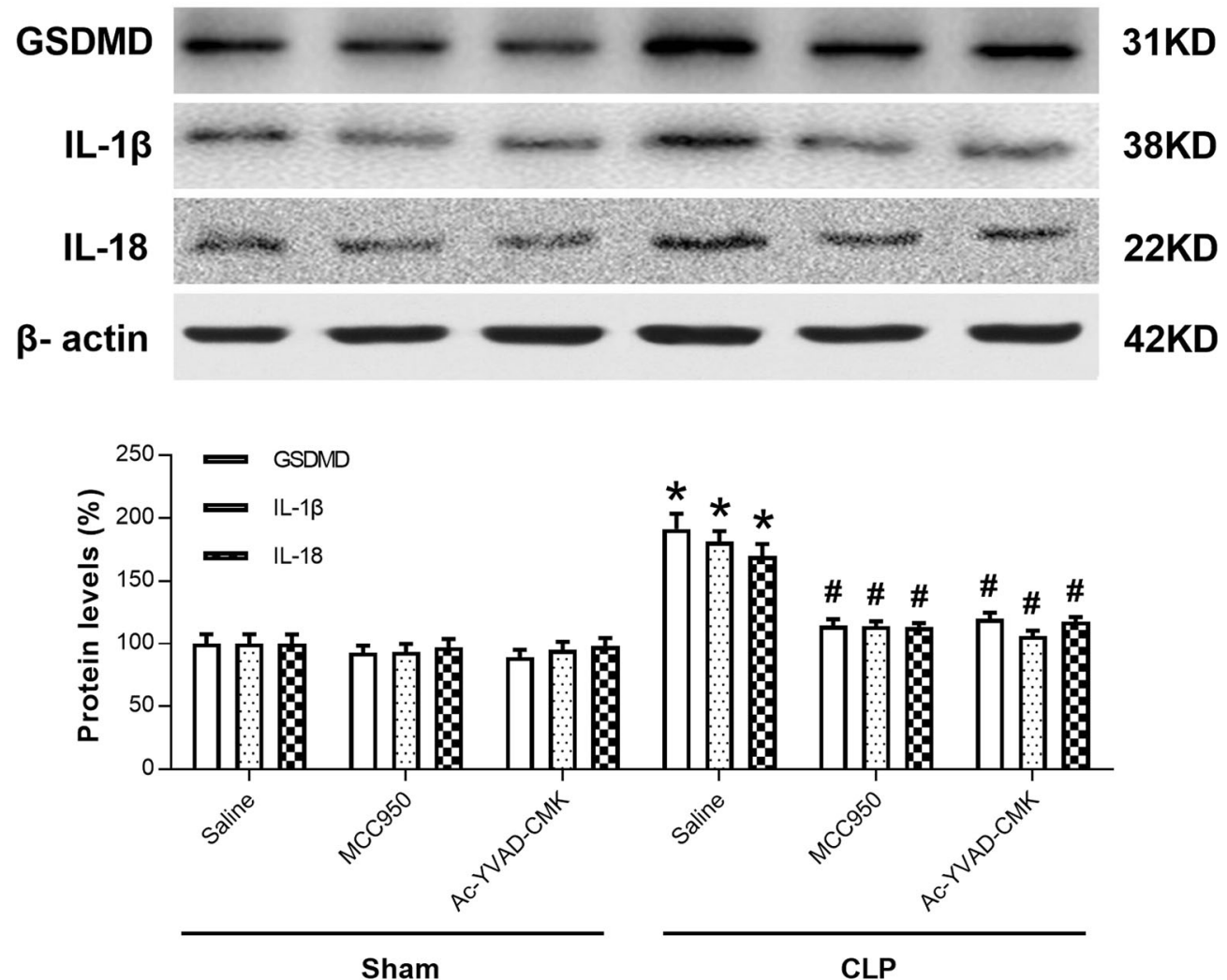

b

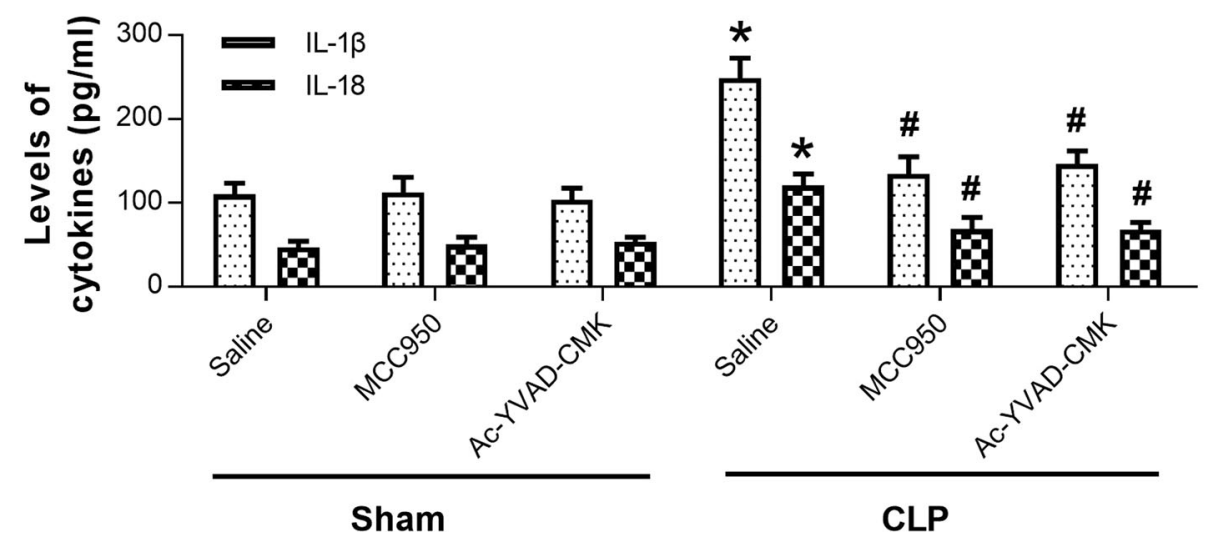

Fig. 7. MCC950 or Ac-YVAD-CMK inhibited NLRP3/caspase-1-dependent pyroptosis and inflammatory cytokines in the hippocampus of SAE mice. a Representative Western blot and quantitative analysis of protein levels of GSDMD, IL- $1 \beta$, and IL- 18 in hippocampal tissues. b ELISA assays of IL- $1 \beta$ and IL-18 levels. Data are shown as mean \pm SEM ( $n=6$ mice/group). The asterisk indicates $P<0.05$ versus the sham + saline group; the pound sign, $P<0.05$ versus the CLP+saline group.

in mice that have undergone CLP. We further show the underlying mechanism of these effects; namely, the sepsis-induced formation of NLRP3 inflammasome leads to caspase- 1 activation and triggers inflammatory cascades and pyroptosis. This supports the hypothesis that MCC950 and Ac-YVAD-CMK inhibit the NLRP3/ 
caspase-1 pathway, alleviating pyroptosis and inflammation, and thus protecting mice from SAE.

Increasing evidence confirms that the brain can be affected during sepsis development, and that septic patients frequently suffer from cognitive impairments after discharge [3]. CLP appears to be a suitable clinical sepsis model and is an important tool by which to study cognitive impairment and its mechanism after sepsis [30]. Our results demonstrate that CLP decreases freezing time in the behavioral context test $24 \mathrm{~h}$ after training but not in the cue test, suggesting sepsis-induced hippocampus-dependent memory impairment in a mouse model of CLP, consistent with our previous investigation [14]. Our study also showed that sepsis does not induce anxiety-like behaviors in SAE mice, and this might be attributed to their full recovery without infection or motor alterations [19].

Neuroinflammation has been proposed as a possible pathogenic mechanism for SAE with long-term cognitive impairment [31, 32]. NLRP3 inflammasome is the most widely investigated inflammasome. It may activate caspase- 1 leading to the processing and secretion of proinflammatory IL-1 $\beta$ and IL-18, which are implicated in several metabolic and inflammatory diseases $[24,33]$. To date, NLRP3 inflammasome research has focused on the pathogenesis of a number of complex conditions, notably autoinflammation and autoimmune disease, that can be treated with the NLRP3-inhibitor MCC950 or the caspase-1 inhibitor Ac-YVAD-CMK [24-26]. In agreement with this, we found that increases of NLRP3, cleaved caspase-1, IL-1 $\beta$, and IL-18 in the hippocampi of mice after CLP were reversed by MCC 950 and that increases of cleaved caspase-1, IL-1 $\beta$, and IL-18 were reversed by Ac-YVAD-CMK, indicating that CLPinduced activation of the NLRP3/caspase-1 pathway is inhibited by administration of MCC950 or Ac-YVADCMK. Most importantly, MCC950 or Ac-YVAD-CMK treatment prevented sepsis-induced neuronal damage and cognitive deficits in CLP mice, suggesting that the NLRP3/caspase-1 pathway is involved in the neurotoxicity and cognitive impairments observed in SAE. When NLRP3 is activated, sensor proteins oligomerize and recruit the adaptor protein ASC which then binds with caspase-1 to form the NLRP3 inflammasome [15]. Unexpectedly, CLP did not increase the expression of ASC in this model. It is possible that other proteins are involved in the formation of the NLRP3 inflammasome, such as NIMA-related kinases (NEK) [34] and protein kinase D (PKD) [35].
Pyroptosis is an inflammatory form of programmed cell death and thought to be involved in neuronal death in the hippocampus, an area of the brain important for learning and memory, leading to cognitive impairments [36]. Excessive pyroptosis causes sepsis and septic shock [27, 37]. Recent advances demonstrate that pyroptotic cell death is mediated by the NLRP3 inflammasome-caspase-1 pathway and that MCC950 and Ac-YVAD-CMK can inhibit the NLRP3 inflammasome response to prevent further pyroptosis [24-26]. GSDMD is the pivotal substrate of pyroptosis in sepsis [27, 37]. In this study, we show that the hippocampus of mice surviving CLP had higher GSDMD expressions than sham-operated groups and that the increase of GSDMD was reversed by administration of MCC950 or Ac-YVAD-CMK, providing further support for the role of pyroptosis in the pathogenesis of SAE. GSDMD lyses liposomes and forms pores on cell membranes; this, in turn, activates the NLRP3 inflammasome, driving caspase-1dependent maturation of IL- $1 \beta$, which is released from the cell upon membrane rupture $[28,38,39]$. Thus, we suggest that interactions among these proteins and cytokines tightly regulate pyroptosis and neuroinflammation responses. While little information about this exists, our data show that sepsis-triggered canonical inflammasome depends on the NLRP3/caspase-1 pathway for the maturation and secretion of IL- $1 \beta$ and on GSDMD for the induction of pyroptosis [40]. Notably, restoration of this pathway by administration of MCC950 or Ac-YVAD-CMK could reduce NLRP3mediated overactivation of neuronal pyroptosis, downregulate the expression of mature IL-1 $\beta$ and IL-18, rescue neuronal damage, and reduce cognitive impairments in SAE mice.

Some limitations must be acknowledged in this study. First, no other specific biomarkers for neuronal pyroptosis in SAE were tested. Second, our experimental model only considers 14 days after CLP, based on a previous study [31], and long-term effects have not yet been considered. Finally, only five administration time points were used and no dose-response study for MCC950 or Ac-YVAD-CMK exists.

In summary, our data suggest the NLRP3/caspase-1 signaling pathway may play a vital role in neuronal pyroptosis and cognitive impairments in the development of SAE secondary to a sepsis. Restoration of the signaling pathway could reverse neurobehavioral abnormities. Inhibition of NLRP3 or caspase-1 provides one possible strategy to prevent and treat SAE. 


\section{FUNDING INFORMATION}

This work was supported by grants from the National Natural Science Foundation of China (Nos. 81471105 and 81571083).

\section{COMPLIANCE WITH ETHICAL STANDARDS}

Conflict of Interest. The authors declare that they have no conflict of interest.

Open Access This article is distributed under the terms of the Creative Commons Attribution 4.0 International License (http://creativecommons.org/licenses/by/4.0/), which permits unrestricted use, distribution, and reproduction in any medium, provided you give appropriate credit to the original author(s) and the source, provide a link to the Creative Commons license, and indicate if changes were made.

\section{REFERENCES}

1. Ji, M.H., L.L. Qiu, H. Tang, L.S. Ju, X.R. Sun, H. Zhang, M. Jia, Z.Y. Zuo, J.C. Shen, and J.J. Yang. 2015. Sepsis-induced selective parvalbumin interneuron phenotype loss and cognitive impairments may be mediated by NADPH oxidase 2 activation in mice. Journal of Neuroinflammation 12: 182.

2. Gofton, T.E., and G.B. Young. 2012. Sepsis-associated encephalopathy. Nature Reviews. Neurology 8: 557-566.

3. Iwashyna, T.J., E.W. Ely, D.M. Smith, and K.M. Langa. 2010. Long-term cognitive impairment and functional disability among survivors of severe sepsis. Journal of the American Medical Association 304: 1787-1794.

4. Mina, F., C.M. Comim, D. Dominguini, O.J. Cassol-Jr, D.M. Dall Igna, G.K. Ferreira, et al. 2014. Il1-beta involvement in cognitive impairment after sepsis. Molecular Neurobiology 49: 1069-1076.

5. Chaudhry, N., and A.K. Duggal. 2014. Sepsis associated encephalopathy. Advance Medical. 2014: 762320.

6. Michels, M., A.S. Vieira, F. Vuolo, H.G. Zapelini, B. Mendonca, F. Mina, et al. 2015. The role of microglia activation in the development of sepsis-induced long-term cognitive impairment. Brain, Behavior, and Immunity 43: 54-59.

7. Jorgensen, I., and E.A. Miao. 2015. Pyroptotic cell death defends against intracellular pathogens. Immunological Reviews 265: 130-142.

8. Kayagaki, N., M.T. Wong, I.B. Stowe, S.R. Ramani, L.C. Gonzalez, S. Akashi-Takamura, K. Miyake, J. Zhang, W.P. Lee, A. Muszynski, L.S. Forsberg, R.W. Carlson, and V.M. Dixit. 2013. Noncanonical inflammasome activation by intracellular LPS independent of TLR4. Science 341: 1246-1249.

9. Kayagaki, N., S. Warming, M. Lamkanfi, L. Vande Walle, S. Louie, J. Dong, et al. 2011. Non-canonical inflammasome activation targets caspase-11. Nature 479: 117-121.
10. Broz, P., and V.M. Dixit. 2016. Inflammasomes: mechanism of assembly, regulation and signalling. Nature Reviews. Immunology 16: 407-420.

11. Qiu, S., J. Liu, and F. Xing. 2017. 'Hints' in the killer protein gasdermin D: unveiling the secrets of gasdermins driving cell death. Cell Death and Differentiation 24: 588-596.

12. Schroder, K., and J. Tschopp. 2010. The inflammasomes. Cell 140: 821-832.

13. Takeuchi, O., and S. Akira. 2010. Pattern recognition receptors and inflammation. Cell 140: 805-820.

14. Wu, J., M. Zhang, S. Hao, M. Jia, M. Ji, L. Qiu, X. Sun, J. Yang, and K. Li. 2015. Mitochondria-targeted peptide reverses mitochondrial dysfunction and cognitive deficits in sepsis-associated encephalopathy. Molecular Neurobiology 52: 783-791.

15. Coll, R.C., A.A. Robertson, J.J. Chae, S.C. Higgins, R. MunozPlanillo, M.C. Inserra, et al. 2015. A small-molecule inhibitor of the NLRP3 inflammasome for the treatment of inflammatory diseases. Nature Medicine 21: 248-255.

16. Chen, Y.L., G. Xu, X. Liang, J. Wei, J. Luo, G.N. Chen, X.D. Yan, X.P. Wen, M. Zhong, and X. Lv. 2016. Inhibition of hepatic cells pyroptosis attenuates CLP-induced acute liver injury. American Journal of Translational Research 8: 5685-5695.

17. Tang, Y., X. Liu, J. Zhao, X. Tan, B. Liu, G. Zhang, L. Sun, D. Han, H. Chen, and M. Wang. 2016. Hypothermia-induced ischemic tolerance is associated with Drp1 inhibition in cerebral ischemiareperfusion injury of mice. Brain Research 1646: 73-83.

18. Gao, R., M.H. Ji, D.P. Gao, R.H. Yang, S.G. Zhang, J.J. Yang, and J.C. Shen. 2017. Neuroinflammation-induced downregulation of hippocampacal neuregulin 1-ErbB4 signaling in the parvalbumin interneurons might contribute to $\operatorname{cog}$ nitive impairment in a mouse model of sepsis-associated encephalopathy. Inflammation 40: 387-400.

19. Comim CM, Cassol OJ, Jr., Abreu I, Moraz T, Constantino LS, Vuolo F, Galant LS, de Rochi N, dos Santos Morais MO, Scaini G, Barichello T, Streck EL, Quevedo J, Dal-Pizzol F Erythropoietin reverts cognitive impairment and alters the oxidative parameters and energetic metabolism in sepsis animal model. Journal of Neural Transmission (Vienna) 2012;119:1267-1274.

20. Heneka, M.T., M.P. Kummer, and E. Latz. 2014. Innate immune activation in neurodegenerative disease. Nature Reviews. Immunology 14: 463-477.

21. Gustin, A., M. Kirchmeyer, E. Koncina, P. Felten, S. Losciuto, T. Heurtaux, A. Tardivel, P. Heuschling, and C. Dostert. 2015. NLRP3 inflammasome is expressed and functional in mouse brain microglia but not in astrocytes. PLoS One 10: e0130624.

22. Heneka, M.T., M.P. Kummer, A. Stutz, A. Delekate, S. Schwartz, A. Vieira-Saecker, A. Griep, D. Axt, A. Remus, T.C. Tzeng, E. Gelpi, A. Halle, M. Korte, E. Latz, and D.T. Golenbock. 2013. NLRP3 is activated in Alzheimer's disease and contributes to pathology in APP/PS1 mice. Nature 493: 674-678.

23. Gross, O., C.J. Thomas, G. Guarda, and J. Tschopp. 2011. The inflammasome: an integrated view. Immunological Reviews 243: 136-151.

24. Jiang, D.L., S. Chen, R.Y. Sun, X. Zhang, and D. Wang. 2018. The NLRP3 inflammasome: role in metabolic disorders and regulation by metabolic pathways. Cancer Letters. 419: 8-19.

25. Dempsey, C., A. Rubio Araiz, K.J. Bryson, O. Finucane, C. Larkin, E.L. Mills, A.A.B. Robertson, M.A. Cooper, L.A.J. O'Neill, and M.A. Lynch. 2017. Inhibiting the NLRP3 inflammasome with MCC950 promotes non-phlogistic clearance of amyloid-beta and cognitive function in APP/PS1 mice. Brain, Behavior, and Immunity 61: 306-316 
26. Wu, D.D., P.H. Pan, B. Liu, X.L. Su, L.M. Zhang, H.Y. Tan, Z. Cao, Z.R. Zhou, H.T. Li, H.S. Li, L. Huang, and Y.Y. Li. 2015. Inhibition of alveolar macrophage pyroptosis reduces lipopolysaccharideinduced acute lung injury in mice. Chinese Medical Journal 128: 2638-2645.

27. Aglietti, R.A., and E.C. Dueber. 2017. Recent insights into the molecular mechanisms underlying pyroptosis and gasdermin family functions. Trends in Immunology 38: 261-271.

28. Shi, J., Y. Zhao, K. Wang, X. Shi, Y. Wang, H. Huang, Y. Zhuang, T. Cai, F. Wang, and F. Shao. 2015. Cleavage of GSDMD by inflammatory caspases determines pyroptotic cell death. Nature 526: 660-665.

29. Shi, J., W. Gao, and F. Shao. 2017. Pyroptosis: gasdermin-mediated programmed necrotic cell death. Trends in Biochemical Sciences 42: 245-254.

30. Granger, J.I., P.L. Ratti, S.C. Datta, R.M. Raymond, and M.R. Opp. 2013. Sepsis-induced morbidity in mice: effects on body temperature, body weight, cage activity, social behavior and cytokines in brain. Psychoneuroendocrinology 38: 1047-1057.

31. Wu, J., L. Dong, M. Zhang, M. Jia, G. Zhang, L. Qiu, M. Ji, and J. Yang. 2013. Class I histone deacetylase inhibitor valproic acid reverses cognitive deficits in a mouse model of septic encephalopathy. Neurochemical Research 38: 2440-2449.

32. Sui DM, Xie Q, Yi WJ, Gupta S, Yu XY, Li JB, et al. Resveratrol protects against sepsis-associated encephalopathy and inhibits the NLRP3/IL-1beta axis in microglia. Mediators of Inflammation 2016;2016:1045657.
33. Mangan, M.S.J., E.J. Olhava, W.R. Roush, H.M. Seidel, G.D. Glick, and E. Latz. 2018. Targeting the NLRP3 inflammasome in inflammatory diseases. Nature Reviews. Drug Discovery 17: 588-606.

34. He, Y., M.Y. Zeng, D. Yang, B. Motro, and G. Nunez. 2016. NEK7 is an essential mediator of NLRP3 activation downstream of potassium efflux. Nature 530: 354-357.

35. Zhang, Z., G. Meszaros, W.T. He, Y. Xu, H. de Fatima Magliarelli, L. Mailly, M. Mihlan, Y. Liu, M. Puig Gámez, A. Goginashvili, A. Pasquier, O. Bielska, B. Neven, P. Quartier, R. Aebersold, T.F. Baumert, P. Georgel, J. Han, and R. Ricci. 2017. Protein kinase D at the Golgi controls NLRP3 inflammasome activation. The Journal of Experimental Medicine 214: 2671-2693.

36. Tan, M.S., L. Tan, T. Jiang, X.C. Zhu, H.F. Wang, C.D. Jia, and J.T. Yu. 2014. Amyloid-beta induces NLRP1-dependent neuronal pyroptosis in models of Alzheimer's disease. Cell Death \& Disease 5: 1382.

37. Gao, Y.L., J.H. Zhai, and Y.F. Chai. 2018. Recent advances in the molecular mechanisms underlying pyroptosis in sepsis. Mediators of Inflammation 2018: 5823823.

38. Ding, J., K. Wang, W. Liu, Y. She, Q. Sun, J. Shi, H. Sun, D.C. Wang, and F. Shao. 2016. Pore-forming activity and structural autoinhibition of the gasdermin family. Nature 535: 111-116.

39. Wallach, D., T.B. Kang, C.P. Dillon, and D.R. Green. 2016. Programmed necrosis in inflammation: toward identification of the effector molecules. Science 352: aaf2154.

40. Rathinam, V.A., and K.A. Fitzgerald. 2016. Inflammasome complexes: emerging mechanisms and effector functions. Cell 165: 792-800. 